\title{
El aprendizaje del tiempo en estudiantes de Educación Secundaria: resultados de rendimiento máximo
}

\author{
The learning of time in students of Secondary Education: results of maximum \\ performance
}

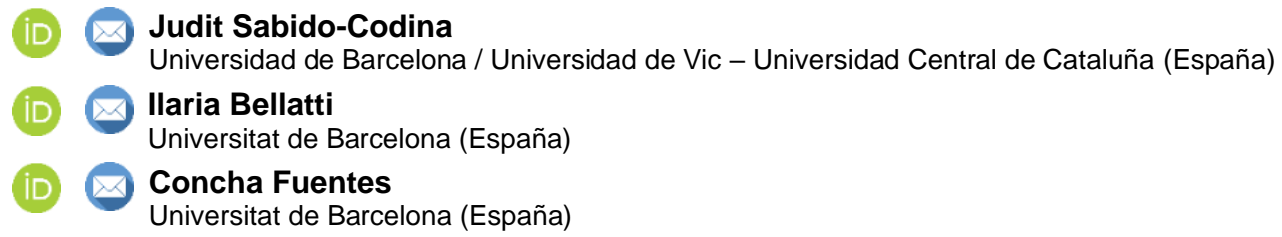

\section{Resumen}

El aprendizaje del tiempo es uno de los grandes retos a los que se ha enfrentado la didáctica de la historia. Muchas investigaciones han esbozado las dificultades que implica su aprendizaje entre el alumnado intentando identificar las nociones temporales más complejas y proporcionando instrumentos didácticos para las aulas. En esta línea, el articulo recoge la investigación titulada El tiempo como núcleo base de la construcción conceptual del pensamiento histórico centrada en analizar los niveles de rendimiento máximo que presentan una muestra de estudiantes de Educación Secundaria y Bachillerato sobre la comprensión de la temporalidad. Para ello, se elaboró una prueba conceptual donde se instaba a los estudiantes a reflexionar sobre los operadores temporales básicos en relación con la datación, la sucesión, la periodización, el cambio y la continuidad, la permanencia y la secuenciación, entre otros. Los resultados indican las dificultades que se perfilan entre los y las estudiantes a la hora de dimensionar determinadas categorías temporales y de relacionarlas correctamente con los hechos históricos de referencia. En las conclusiones se señala la necesidad de trabajar los operadores y categorías temporales de los hechos históricos, considerados factuum aislados, en combinación con su devenir en la narrativa y explicación causal entendida como continuum.

\begin{abstract}
The learning of time is one of the great challenges that the didactics of history has faced. Many investigations have outlined the difficulties involved in their learning among students, trying to identify the most complex temporal notions and providing didactic tools for classrooms. In this line, the article collects the research entitled Time as the base nucleus of the conceptual construction of historical thought focused on analyzing the maximum performance levels presented by a sample of Secondary Education and Baccalaureate students on the understanding of temporality. To do this, the starting point was the elaboration of a conceptual test where students were urged to reflect on the basic temporal operators in relation to dating, succession, periodization, change and continuity, permanence, and sequencing, among others. The results indicate the difficulties that are outlined among the students when it comes to dimensioning certain temporal categories and correctly relating them to the historical facts of reference. The conclusions indicate the need to work on the temporal operators and categories of historical events, considered as isolated factuum, with their evolution in the narrative and causal explanation as a continuum.
\end{abstract}

\section{Palabras clave / Keywords}

tiempo histórico, enseñanza de las ciencias sociales, educación secundaria, evaluación comparativa.

Historical Time, Social Science Education, Secondary Education, Comparative Evaluation. 


\section{Introducción}

El aprendizaje del tiempo histórico ha supuesto desde los inicios de la didáctica de las ciencias sociales un gran reto para los investigadores centrados en analizar la enseñanza de la historia (Torres, 2001; Blanco, 2007; Díaz-Barriga et al, 2008; Llusà, 2015; Miralles y Gómez, 2016; Santiesteban, 2017; ). La dificultad que implica la adquisición de las nociones temporales, desde la primera infancia hasta la educación secundaria, ha sido una preocupación que ha entrelazado la investigación de la didáctica de la historia con la de la psicología cognitiva y social, y ejemplo de ello son las investigaciones de Jacott (1995), Carretero y Montaner (2008), entre otros.

En primera instancia, los intereses de los autores se han centrado en identificar aquellas categorías y nociones temporales propias del quehacer histórico en relación con la forma de abordar el tiempo en el aula (Duquette, 2011; Seixas y Morton, 2012), considerando el aprendizaje temporal como elemento fundamental para la construcción del conocimiento histórico. Por otro lado, se ha investigado la forma cómo estas categorías funcionan en la comprensión temporal de niños y adolescentes (Torres, 2001; Trepat, 2002; De GrootReuvekamp, Ros y Van-Boxtel, 2017; Birkeland, 2018; Solé, 2019; Pinto y Pagès, 2020;). Un punto de inflexión en las investigaciones realizadas en España lo alcanza Santisteban (2017) cuando, en una revisión del estado de la cuestión reconduce dos líneas que se habían desarrollado de forma separada: las investigaciones sobre aprendizaje y comprensión del tiempo histórico, en su vertiente más disciplinarhistórica, y las investigaciones sobre formación del pensamiento histórico y su aprendizaje dentro del paradigma de conciencia histórica y temporal. Siguiendo a esta última corriente, el aprendizaje del tiempo histórico se relaciona con el desarrollo del lenguaje y con la construcción narrativa causal de los hallazgos históricos. De esta forma, se empieza a plantear parte del proceso de aprendizaje de la materia aplicando una didáctica del tiempo adecuada a los niveles de progresión y en acuerdo con el método histórico. En esta línea, nuestro estudio se propone comprobar la operatividad de categorías temporales, en términos de rendimiento máximo, creadas en relación con investigaciones previas como la de Blanco (2007). A la vez, pretende comprender y contextualizar la eficacia de este aprendizaje del tiempo en la reconstrucción narrativa de los discentes del hecho histórico, factuum, que, siguiendo a autores como Hammond (2014) o De la Paz et al (2017) influye directamente en comprender la historia como un continuum, es decir como un devenir interconectado de acontecimientos históricos.

\subsection{El tiempo histórico y sus operadores temporales}

El tiempo histórico, como constructo social propio de la disciplina y de la narrativa histórica, conlleva dificultades congénitas sobre su medición y percepción. Dificultades que hoy en día siguen presentes en los debates académicos (Tamm y Olivier, 2019). Los acontecimientos históricos, en relación con su tratamiento, pueden ser organizados como un factuum, que sería el tratamiento de cada hecho histórico en sí, o un continuum. Por lo que concierne el continuum, puede ser explicado desde ritmos y duraciones variables. Por ello, decimos que el tiempo histórico se caracteriza por su pluralidad, no necesariamente lineal, y está formado por innumerables niveles temporales que se superponen (Ost, 2000; Buck, 2020) y permiten narraciones de corta, media y larga duración de los eventos históricos. Diversos autores ven el pensamiento temporal como un conjunto de relaciones conceptuales que confeccionan una red de estructuras temporales a través de las cuales se construye la narrativa histórica en diferentes niveles (Assmann, 2019). A dichas estructuras Matozzi las denominó operadores temporales cognitivos (Mattozzi, 1988; 2004). Es decir, que el tiempo histórico puede ser aprendido por los alumnos trabajando estos operadores temporales de forma separada. Este análisis se puede realizar en relación con el factuum histórico (las categorías más cronológicas y lineales), y en relación con la narrativa histórica (categorías que permiten crear explicaciones causales más interconectadas, multinivel entre diferentes hechos históricos ubicados en tiempo y espacios diversos), para ejercitar en alumnos habilidades temporales y, en definitiva, procurar un acercamiento a la historia mucho más significativo. Esta red de estructuras temporales se ha ido delimitando en diversos estudios producidos desde el ámbito específico de la didáctica de las ciencias sociales (Blanco, 2007; Blanco, 2008; Sáiz, 2013; Sáiz y Colomer, 2014; López y Llull, 2017), en los cuales, por lo general, se han considerado como subestructuras temporales el cambio, la continuidad, la duración, la causalidad, la periodización, la datación, la permanencia, la secuenciación, la sucesión y la simultaneidad. 


\subsection{El aprendizaje del tiempo entre dificultades y retos}

Las dificultades a las que se enfrenta la didáctica del tiempo, tanto a nivel de investigación, como de evaluación de los aprendizajes, se podrían concentrar alrededor de dos aspectos. En primer lugar, la percepción del tiempo como cambio y, en segundo lugar, una comprensión más conceptual relacionada con el proceso de significación que abarca desde la aproximación temporal de acontecimientos históricos alejados en el tiempo, a su relación con el entorno próximo del alumnado (Llusà, 2015; Miralles y Gómez, 2016; De Groot-Reuvekamp, Ros y Van-Boxtel, 2017; Solé, 2019; Pinto y Pagès, 2020). Dicho de otra forma, el problema no se da solo al tratar temas de un periodo histórico intangible a los ojos de niños y adolescentes, sino también a la hora de buscar lazos significativos con el presente, motivando la comprensión multicausal de las explicaciones históricas en edades tempranas (Lee y Shemilt, 2004; Wilschut, 2019; Keynes, 2021). El proceso que conlleva el aprendizaje del tiempo histórico desde esta visión más holística, según la cual se estudian los significados de los procesos históricos, a la vez que se ubican temporalmente y se elaboran explicaciones causales, es obstaculizado por la secuenciación lineal y, aparentemente ordenada, de los contenidos organizados en los libros de texto según las convenciones temporales de las periodizaciones clásicas: prehistoria, historia antigua, medieval, moderna, contemporánea e historia reciente (Bellatti, 2019). Es decir, que, si se utilizan los libros de texto como únicos recursos para el aprendizaje temporal, se corre el riesgo de reducir la complejidad de la temporalización histórica a la de secuenciación, dejando totalmente excluidas relaciones temporales como el de la simultaneidad, y simplificando al máximo las relaciones entre cambio y continuidad (Bellatti y Sabido-Codina, 2017; López y Llull, 2017). Es por este motivo, por el que las múltiples investigaciones relacionadas con el libro de texto y currículos educativos han intentado jerarquizar los distintos operadores temporales en relación con su dificultad, y han tratado de pautar su aplicación progresiva para optimizar el aprendizaje de la historia (Torres, 2001; Trepat, 2002; Blanco, 2008).

\section{Objetivos}

El estudio que presentamos emerge de los resultados de una investigación titulada "El tiempo como núcleo base de la construcción conceptual del pensamiento histórico". El objetivo fundamental consistía en analizar los niveles de rendimiento máximo de estudiantes de $4^{\circ}$ de la ESO y $2^{\circ}$ de Bachillerato por lo que respecta al uso aplicado de los operadores temporales prefijados, en relación con su comprensión del hecho histórico como factuum y como continuum. Como objetivos específicos nos planteamos:

1. Elaborar una prueba conceptual que trabaje los diversos operadores temporales en relación con su comprensión del hecho histórico, como factuum y como continuum.

2. Determinar matemáticamente los niveles de rendimiento máximo de los operadores temporales en relación con una prueba conceptual.

3. Comparar los niveles de rendimiento máximo entre estudiantes de $4^{\circ}$ de la ESO y $2^{\circ}$ de Bachillerato.

Con ello, pretendemos generar una discusión con las investigaciones previas, ya sea tanto desde una perspectiva psicológica como desde una perspectiva la didáctica, sobre la aplicabilidad de los distintos operadores temporales. De esta forma, se pretende dilucidar si realmente se puede hablar de los diferentes niveles de dificultad entre los operadores temporales citados.

\section{Metodología}

Para alcanzar el objetivo general y los objetivos específicos del presente estudio se ha optado por utilizar una metodología cuantitativa-mixta. El instrumento de investigación diseñado se ha basado en una prueba de conocimiento, donde se han incluido preguntas de diferente naturaleza: abiertas y mixtas. En este artículo presentamos los resultados de las preguntas del número 3 al número 10. Asimismo, cabe destacar que, siguiendo la línea de Strauss y Corbin (2002), se han incorporado elementos de reflexividad constante, propiciando el análisis en diferentes momentos de la investigación. Es por ese motivo, que el instrumento de investigación diseñado es de naturaleza cognitivo-construccionista (Sánchez-Carrión, 2012). 


\subsection{La muestra}

Se ha optado por un muestreo intencional criterial. Una muestra no probabilística de representatividad subjetiva (Scharager y Armijo, 2001). Esta tipología de muestra, al igual que la probabilística estratificada, escoge casos concretos agrupados por características específicas predeterminadas en acuerdo a las necesidades propias del estudio (López, 2004).

Los criterios usados han sido dos. Por un lado, se ha limitado la muestra geográficamente, por tanto, todos los individuos seleccionados, agrupados en centros educativos de educación obligatoria y postobligatoria, son de la Comunidad Autónoma de Cataluña. Por otro lado, se han seleccionados los centros educativos en relación con la situación sociodemográfica ${ }^{1}$ para generar indicadores educativos de éxito.

En total se han seleccionado 6 centros educativos de la Comunidad Autónoma de Cataluña $(n=213)$, dos de los cuales tienen una situación óptima para generar indicadores educativos de éxitos, otros dos tienen una situación moderada, y los dos últimos tienen una situación compleja. Concretamente se ha pasado el instrumento de investigación a 154 estudiantes de $4^{\circ}$ de ESO y 59 de 2ํㅡㄹ de Bachillerato (ver Gráfico 1).

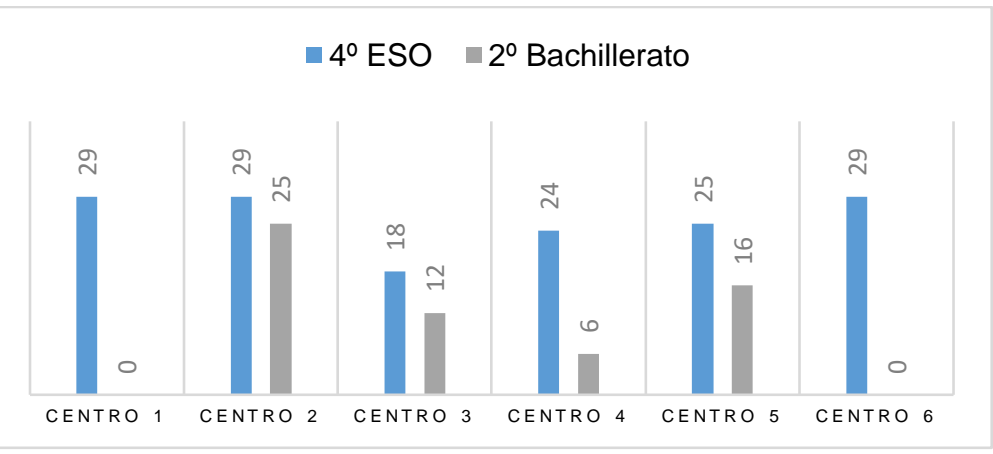

\section{Gráfico 1. Delimitación de la muestra a partir de los centros analizados}

\subsection{El instrumento de investigación}

El instrumento de investigación fue confeccionado a partir de cuatro fases de validación. En una primera fase (Fase I) se realizó una sucesión de actividades que trabajaban las distintas categorías temporales, éstas pasaron por una comisión de expertos y fueron matizadas.

El primer esbozo del instrumento, Fase II, fue pre-pilotado $(n=60)$ en dos centros educativos. Este primer contacto se realizó únicamente con estudiantes de 4ํㅡㄹ de la ESO y permitió matizar las distintas preguntas del instrumento, así como ver la idoneidad de estas en relación con su objetivo de investigación.

A continuación, Fase III, se procedió al pilotaje del instrumento $(n=42)$, y se comprobó su idoneidad con estudiantes de $4^{\circ}$ de la ESO y de $2^{\circ}$ de Bachillerato. La principal divergencia entre unos y otros era la comprensión lectora. Como resultado, la mayoría de estudiantes de 4ํ de la ESO analizados no terminaban la prueba. Es por eso, que se generaron dos modelos de prueba, la A y la B, donde se cambiaron el orden de las preguntas para así obtener un número adecuado de respuestas para su futuro análisis.

En una última fase, Fase IV, se realizó el proceso de validación final con varios expertos del ámbito, los cuales acordaron un nivel de acuerdo alto, por lo que se refiere a la relación entre las preguntas del instrumento y los objetivos generales de análisis, así como su descriptor (ver Tabla. 1).

\footnotetext{
${ }^{1}$ Se ha considerado seis indicadores para seleccionar cada uno de los centros: 1) bajo nivel de instrucción de padres o madres /tutores o tutoras; 2) ocupación de trabajos de baja calificación profesional de los padres o madres /tutores o tutoras; 3) número significativo de padres/ madres o tutores/ tutoras de estudiantes perceptores de la renda mínima; 4) porcentaje elevado de padres o madres/ tutores 0 tutoras en situación de paro; 5) alto porcentaje de estudiantes con necesidades específicas; 6) porcentaje alto de estudiantes recién llegados.
} 
Tabla 1

Relación entre preguntas del instrumento, objetivos de análisis y descriptor

\begin{tabular}{|c|c|c|}
\hline $\begin{array}{l}\text { Preguntas } \\
\text { instrumento }\end{array}$ & Objetivos generales de análisis & Descriptor \\
\hline Pregunta 3 & $\begin{array}{l}\text { Analizar la datación y } \\
\text { simultaneidad de los sucesos } \\
\text { históricos a partir de } \\
\text { justificaciones lingüísticas }\end{array}$ & $\begin{array}{l}\text { Analizar la datación como identificación de los sucesos históricos a partir de } \\
\text { justificaciones lingüísticas } \\
\text { Analizar la simultaneidad como continuum de la historia }\end{array}$ \\
\hline Pregunta 4 & $\begin{array}{l}\text { Identificar la datación, sucesión, } \\
\text { secuenciación y simultaneidad. }\end{array}$ & $\begin{array}{l}\text { Identificar la datación como delimitación temporal en una línea del tiempo. } \\
\text { Identificar la sucesión como la ordenación de los acontecimientos en una } \\
\text { línea temporal. } \\
\text { Identificar la secuenciación como encuadre de un periodo concreto queafectan } \\
\text { a los periodos posteriores cercanos en el tiempo. } \\
\text { Identificar la simultaneidad como yuxtaposición de un hecho que transcurreen } \\
\text { el mismo tiempo. }\end{array}$ \\
\hline Pregunta 5 & $\begin{array}{l}\text { Analizar la interpretación de las } \\
\text { duraciones a partir de la } \\
\text { justificación lingüística. }\end{array}$ & $\begin{array}{l}\text { Analizar la duración como resultado de una repetición especifica de } \\
\text { acontecimientos y/o años } \\
\text { Analizar el cambio y la continuidad a partir de la duración } \\
\text { Analizar la simultaneidad como perspectiva sobre la inmediatez } \\
\text { Analizar la causalidad como resultado de una duración }\end{array}$ \\
\hline Pregunta 6 & $\begin{array}{l}\text { Identificar las periodizaciones a } \\
\text { partir de un recurso iconográfico y } \\
\text { una datación exacta. }\end{array}$ & $\begin{array}{l}\text { Identificar la periodización como regulador temporal de la periodización } \\
\text { tradicional europea }\end{array}$ \\
\hline Pregunta 7 & $\begin{array}{l}\text { Identificar las sucesiones } \\
\text { históricas correctas e incorrectas. }\end{array}$ & $\begin{array}{l}\text { Identificar la sucesión como la ordenación de los acontecimientos a partir de } \\
\text { un antes y después, de forma progresiva }\end{array}$ \\
\hline Pregunta 8 & $\begin{array}{l}\text { Analizar los distintos ritmos de } \\
\text { cambio y continuidad a partir de } \\
\text { los mapas históricos. }\end{array}$ & $\begin{array}{l}\text { Analizar el cambio y la continuidad a partir de la significación de cambio. } \\
\text { Analizar la permanencia como resultado de la expansión de un cambio. } \\
\text { Analizar la causalidad como consecuencia de un cambio. }\end{array}$ \\
\hline Pregunta 9 & $\begin{array}{l}\text { Identificar y justificar las distintas } \\
\text { aceleraciones de cambio de una } \\
\text { ciudad a partir de la narrativa. }\end{array}$ & $\begin{array}{l}\text { Identificar el cambio y la continuidad a partir de la percepción de cambio } \\
\text { coyuntural o a partir de la proporcionalidad de cambio y permanencia. } \\
\text { Identificar la permanencia como duración de un fenómeno pasado. Identificar } \\
\text { la simultaneidad como como percepción de cambio coyuntural. }\end{array}$ \\
\hline Pregunta 10 & $\begin{array}{l}\text { Identificar la tipología de narrativa } \\
\text { histórica elaborada por los } \\
\text { estudiantes y visualizar la } \\
\text { concepción de cambio en ellas. } \\
\text { Identificar si hay una } \\
\text { interpretación crítica del hecho } \\
\text { histórico. }\end{array}$ & $\begin{array}{l}\text { Identificar el cambio y la continuidad a partir de las explicaciones libres de } \\
\text { tópicos. } \\
\text { Identificar la permanencia como continuidad de un fenómeno presente. } \\
\text { Identificar la simultaneidad como continuum de la historia }\end{array}$ \\
\hline
\end{tabular}

Finalmente, se obtuvo una prueba conceptual compuesta por 10 preguntas (ver Figura. 1), 8 de las cuales trabajaban las distintas categorías temporales (el cambio, la continuidad, la duración, la causalidad, la periodización, la datación, la permanencia, la secuenciación, la sucesión y la simultaneidad), mientras que la pregunta 1 y 2 , que no son objeto de análisis en el presente artículo, pretendían analizar la percepción de la temporalidad a partir de una imagen y de un texto.

Las categorías temporales seleccionadas son las mismas que utilizó Blanco en su tesis doctoral (2007), no obstante, en dicha investigación no se ha contemplado la empatía histórica, ya que la vemos como un resultado del aprendizaje del tiempo y no como un operador.

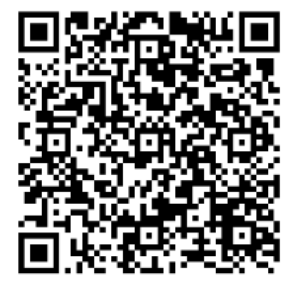

Figura 1. Instrumento de investigación

\subsection{Categorías de análisis y fiabilidad}


Los datos se transcribieron y se vaciaron en una matriz de información de Excel. Seguidamente se determinó un valor de correcto o incorrecto a todas las preguntas del instrumento, tanto las cerradas como las mixtas (cerradas y abiertas). Después de ello, se categorizaron las preguntas abiertas que suponían una justificación narrativa sobre la importancia del hecho histórico, en termino de significación histórica, para estimular explicaciones causales-temporales (ver Tabla 2). Esta explicación eliminaba la aleatoriedad en el asertividad de la pregunta. Por tanto, solo se consideraban como correctas aquellas preguntas abiertas que estaban justificadas por una narrativa adecuada.

\section{Tabla 2}

Relación entre las preguntas y las categorías preestablecidas

\begin{tabular}{|c|c|c|}
\hline Pregunta & ategorías & Ejemplo \\
\hline \multirow[b]{2}{*}{3 y 10} & Explicación cronológica: factuum & רo 1789 , es decir, \\
\hline & Explicación conceptual: continuum & $\begin{array}{l}\text { "Ambos hechos fueron de gran importancia. El primero inauguró nuevos estadios } \\
\text { nacionales y la caída del Imperio romano occidental, también este hecho } \\
\text { ocasionaría la expansión del Imperio otomano" [A.6.06.E] }\end{array}$ \\
\hline \multirow{3}{*}{5} & Perspectiva cronológica: factuum & $\begin{array}{l}\text { "El descubrimiento de América es corta duración, porque es un hecho de un año } \\
\text { concreto" [A.1.19.E] }\end{array}$ \\
\hline & tiva inmediatez: continuum & $\begin{array}{l}\text { "El descubrimiento de América es larga duración, porque los españoles } \\
\text { perduraron ahí durante siglos explotando los terrenos, cometiendo genocidios y } \\
\text { esclavizando" [A.6.18.E] }\end{array}$ \\
\hline & Cambio con visión cronológica & $\begin{array}{l}\text { "En el primer mapa se trata de un período de algo menos de } 3 \text { años y en el } \\
\text { segundo son siglos" [A.2.16.B] } \\
\text { "La diferencia es abismal porque el Imperio Romano no solo conquistaba pocos }\end{array}$ \\
\hline \multirow[t]{4}{*}{8} & Cambio territorial & $\begin{array}{l}\text { sitios, sino que conquistó casi toda Europa y una pequeña parte de África, en } \\
\text { cambio Franco solo pudo con una parte de país, que fue España" [A.4.07.E] }\end{array}$ \\
\hline & Cambio tecnológico & "La Guerra Civil fue más eficaz, ya que contaban con una artillería más novedosa" \\
\hline & Cambio social & $\begin{array}{l}\text { "A partir de casi } 1.500 \text { años hay diferencias en todo, excepto en la geografía física. } \\
\text { Las otras lenguas, otras culturas, otros medios de producción, otras ideologías, } \\
\text { un mundo distinto" [A.5.06.B] }\end{array}$ \\
\hline & ión de coyuntura & $\begin{array}{l}\text { "Del texto B al C hay } 100 \text { años. En el C se introducen productos americanos, } \\
\text { aunque no se habla de la revolución industrial" [A.6.19.E] }\end{array}$ \\
\hline $\begin{array}{l}\text { Pregunta } \\
9\end{array}$ & $\begin{array}{l}\text { Percepción aproximada de la } \\
\text { proporcionalidad de cambios y } \\
\text { permanencia }\end{array}$ & $\begin{array}{l}\text { "Del texto A al B va del s. XIV - s. X VI al s. XVII-XVIII, porque la pes } \\
\text { entre el s. XIV-s. XVI y la burguesía en el s. XVII-s. XVIII" [A.1.21.E] }\end{array}$ \\
\hline
\end{tabular}

Cabe remarcar que, para garantizar la estabilidad, la consistencia y la dependencia de los datos se realizó un control de fiabilidad entre distintos expertos del ámbito, calculando un alfa de Krippendorff ${ }^{2}$. Se comprueba la concordancia entre codificadores y se determina si han llegado a una misma decisión (Torres y Perera, 2010). Es por ello, que dos expertos del área más la investigadora codificaron, por un lado y por lo que respecta a las preguntas abiertas, un total de 19 casos con sus respectivas categorías previamente definidas y realizaron 57 decisiones. Y por otro lado, por lo que respecta a las preguntas cerradas, codificaron un total de 11 casos y realizaron 33 decisiones. Las preguntas abiertas obtuvieron un alfa de Krippendorff de 0.85 (nominal) y las preguntas cerradas un alfa de Krippendorff de 0.99 (ordinal), por tanto, ambas codificaciones obtuvieron una fiabilidad alta de codificación (Löfgren, 2017).

\section{Resultados}

El instrumento de investigación recogió la presencia de cada una de las 10 categorías citadas. Los resultados del análisis de los datos $\left(n=213 ; 4^{\circ}\right.$ de la ESO $n=154$ y $2^{\circ}$ de Bachillerato $\left.n=59\right)$, específicamente de la pregunta 3 a la 10 nos dan una visión concreta de los conocimientos que presentan los y las estudiantes sobre dichas variables. Cabe remarcar, que en el momento que se pasó el instrumento, los y las estudiantes ya habían trabajado todos los hechos históricos que salen en él. Asimismo, y siguiendo las directrices marcadas por el curriculum, por lo que respecta al uso de las categorías temporales, los y las estudiantes deberían ya tener en esa etapa educativa un óptimo desarrollo de todas ellas. Del vaciado de las diversas preguntas, destaca el elevado porcentaje de las preguntas parcialmente sin responder y las justificaciones sin argumento. No obstante, si observamos las respuestas proporcionadas de

\footnotetext{
${ }^{2}$ Para el cálculo del alfa de Krippendorff se utilizo el recurso en línea ReCal (http://dfreelon.org/recal/recal-oir.php\#result1).
} 
forma global, es decir contemplando aquellas que han sido respuestas a pesar del modo o del texto escrito, se calcula una tasa apreciable de respuesta adecuada (ver Tabla 3).

Tabla 3

Tasa de respuesta de las preguntas del instrumento de investigación

\begin{tabular}{lll}
\hline & $\%$ \% & $\%$ \\
& $4^{0}$ ESO & $2^{0}$ Bachillerato \\
\hline Pregunta 3 & $87,3 \%$ & $93 \%$ \\
Pregunta 4 & $52 \%$ & $81,4 \%$ \\
Pregunta 5 & $53,9 \%$ & $88 \%$ \\
Pregunta 6 & $64,3 \%$ & $95 \%$ \\
Pregunta 7 & $77,2 \%$ & $96,6 \%$ \\
Pregunta 8 & $93,75 \%$ & $96,5 \%$ \\
Pregunta 9 & $62,3 \%$ & $79,7 \%$ \\
Pregunta 10 & $74,7 \%$ & $81 \%$
\end{tabular}

A continuación, vamos a indicar los resultados obtenidos por cada pregunta diseñada para el ejercicio y la comprobación de la adquisición de cada uno de los operadores temporales (ver Tabla 1). Cabe remarcar que los intervalos matemáticos que definirán el nivel de rendimiento máximo son [<20\% "muy bajo"], [21\%- 40\% "bajo"], [41\%-60\% "moderado"], [61\%-80\% "bueno"] y [81\%-100\% "muy bueno"].

\subsection{La datación}

La datación se ha analizado a partir de dos descriptores (ver Tabla 1): uno por la pregunta 3 y otro por la pregunta 4. Los resultados obtenidos (ver Tabla 4) en la pregunta 4 manifiestan que los y las estudiantes presentan problemas a la hora de situar los diversos hechos históricos en una línea temporal, sobre todo por lo que respecta aquellos hechos antes de Cristo. Asimismo, vemos como pocos identifican adecuadamente los intervalos de años presentados en la pregunta 3 con su respectivo hecho histórico.

\section{Tabla 4}

Nivel de rendimiento máximo: datación

\begin{tabular}{lllll}
\hline & Pregunta.3 & Pregunta.4 & Media entre P.3 y P.4 & Nivel de rendimiento máximo esperado \\
\hline $4^{\circ}$ de ESO & $4,6 \%$ & $15,6 \%$ & $10,1 \%$ & Muy bajo \\
$2^{\circ}$ de Bachillerato & $10,2 \%$ & $49,2 \%$ & $29 \%$ & Bajo \\
\hline
\end{tabular}

\subsection{La sucesión}

La sucesión (ver Tabla 5) al igual que la datación se ha analizado a partir de dos descriptores (ver Tabla 1): uno por la pregunta 4 y otro por la pregunta 7 . El análisis de la pregunta 3 ha reflejado como 3 de cada 10 estudiantes en $4^{\circ}$ de la ESO, y 5 de cada 10 estudiantes en $2^{\circ}$ de Bachillerato, han situado correctamente los diversos hechos históricos en términos de sucesión en la línea temporal propuesta. Nuevamente, los principales problemas aparecen en la colocación de los hechos a.C. y d.C. Sin embargo, por lo que respecta los resultados de la pregunta 7, los y las estudiantes no consiguen identificar correctamente si los diversos hechos históricos están ordenados correctamente. Sorprendentemente, destaca que aproximadamente 2 de cada 10 estudiantes en ambos cursos no identifican como correcta la sucesión: Segunda República Española - Guerra Civil - Franquismo. 
Tabla 5

Nivel de rendimiento máximo: sucesión

\begin{tabular}{lllll}
\hline & Pregunta.4 & Pregunta.7 & Media & Nivel de rendimiento máximo esperado \\
\hline $4^{\circ}$ de ESO & $26,6 \%$ & $6,5 \%$ & $16,55 \%$ & Muy bajo \\
2o de Bachillerato & $51 \%$ & $23,7 \%$ & $37,35 \%$ & Bajo \\
\hline
\end{tabular}

\subsection{La periodización}

La periodización se ha analizado a partir de los resultados de la pregunta 6 (ver Tabla 6). Éstos han reflejado que los y las estudiantes tienen un dominio adecuado de la periodización, datos que van en la línea de la investigación de Bellatti (2018) sobre el uso hegemónico de la periodización histórica convencional en los libros de texto. Cabe remarcar que el principal error que han cometido los y las estudiantes de ambos cursos emerge de la confusión entre la Edad Moderna y la Edad Contemporánea.

\section{Tabla 6}

Nivel de rendimiento máximo: periodización

\begin{tabular}{lll}
\hline & Pregunta.6 & Nivel de rendimiento máximo esperado \\
\hline $4^{\circ}$ de ESO & $42,2 \%$ & Moderado \\
$2^{\circ}$ de Bachillerato & $79,7 \%$ & Bueno \\
\hline
\end{tabular}

\subsection{El cambio y la continuidad}

El cambio y la continuidad, al igual que la periodización, ha presentado resultados óptimos por lo que respecta su desempeño. Estos operadores temporales se han analizados en relación con cuatro descriptores. En primer lugar, se ha analizado el cambio y la continuidad a partir de la duración. Este primer análisis ha partido de la pregunta 5 . Los resultados han sido muy divergentes, ya que el $64,4 \%$ de los y las estudiantes de $2^{\circ}$ de Bachillerato han dado una respuesta coherente de cambio y continuidad a partir de la duración, mientras que en $4^{\circ}$ de la ESO solo $28,6 \%$ lo han hecho. En segundo lugar, se ha analizado el cambio y continuidad como significación de cambio (pregunta 8), dando unos resultados muy semejantes a los obtenidos en el descriptor anterior. En tercer lugar, se ha analizado el cambio y continuidad como percepción de cambio coyuntural y como proporcionalidad de cambio y permanencia (pregunta 9). Ésta ha sido la concepción referida al cambio y continuidad que más dificultades ha presentado. En cuarto, y último lugar, se ha analizado el cambio y continuidad de las explicaciones libres de tópicos (pregunta 10). Se aprecia que el 32,5\% los y las estudiantes de $4^{\circ}$ de la ESO han realizado una explicación conceptual cronológica básica y sin tópicos, mientras que los alumnos de $2^{\circ}$ de Bachillerato lo han realizado un $39 \%$ tal y como se aprecia en la tabla 7.

\section{Tabla 7}

Nivel de rendimiento máximo: cambio y continuidad

\begin{tabular}{lllllll}
\hline & Pregunta.5 & Pregunta.8 & Pregunta.9 & Pregunta.10 & Media & Nivel de rendimiento máximo esperado \\
\hline $4^{\circ}$ de ESO & $28,6 \%$ & $39,6 \%$ & $11 \%$ & $32,5 \%$ & $27,9 \%$ & Bajo \\
20 de Bachillerato & $64,4 \%$ & $61 \%$ & $6,8 \%$ & $39 \%$ & $42,8 \%$ & Moderado \\
\hline
\end{tabular}

\subsection{La permanencia}

La permanencia (ver Tabla 8) se ha analizado a partir de tres descriptores. En primer lugar, hemos analizado la permanencia como resultado de la expansión de un cambio (pregunta 8). En segundo lugar, la permanencia entendida como la duración de un fenómeno pasado (pregunta 9). Y en último, lugar, hemos analizado la permanencia como continuidad de un fenómeno presente (pregunta 10). Por lo general, hemos observado como los y las estudiantes identifican mejor la permanencia a través de los mapas, es decir a través de un recurso iconográfico. Muchas más dificultades han presentado aquellas actividades trabajaban la permanencia a través de un recurso textual (pregunta 9) o de un saber pasado (pregunta 10). 
Tabla 8

Nivel de rendimiento máximo: permanencia

\begin{tabular}{llllll}
\hline & Pregunta.8 & Pregunta.9 & Pregunta.10 & Media & Nivel de rendimiento máximo esperado \\
\hline $4^{\circ}$ de ESO & $39,6 \%$ & $11 \%$ & $13,6 \%$ & $21,4 \%$ & Bajo \\
$2^{\circ}$ de Bachillerato & $61 \%$ & $6,8 \%$ & $20,3 \%$ & $29,4 \%$ & Bajo \\
\hline
\end{tabular}

\subsection{La secuenciación}

La secuenciación (ver Tabla 9) se ha analizado a partir de un único descriptor: la secuenciación como encuadre de un periodo concreto que afecta a los periodos posteriores cercanos en una línea del tiempo (pregunta 4). Los y las estudiantes han manifestado dificultades en secuenciar temporalmente hechos de diversa duración: generalmente, encuadraban en un mismo espacio de tiempo tanto períodos de $<6$ años, como por ejemplo la Guerra Civil, como períodos de >100 años, como por ejemplo el imperio romano.

Tabla 9

Nivel de rendimiento máximo: secuenciación

\begin{tabular}{lll}
\hline & Pregunta.4 & Nivel de rendimiento máximo esperado \\
\hline $4^{\circ}$ de ESO & $14,9 \%$ & Muy bajo \\
20 de Bachillerato & $27,2 \%$ & Bajo \\
\hline
\end{tabular}

\subsection{La simultaneidad}

La simultaneidad (ver Tabla 10) se ha analizado en relación con cuatro descriptores. En primer lugar, hemos analizado la simultaneidad como continuum de la historia. En los resultados obtenidos en la pregunta 3 y 10 , se aprecia que los y las estudiantes prefieren justificar su elección a partir de explicaciones cronológicas y no tanto conceptuales. Por lo que respecta a las preguntas 5 y 9 , donde se analizaba la simultaneidad entendida como perspectiva sobre la inmediatez y la simultaneidad como percepción de cambio coyuntura, vemos que son muy pocos los y las estudiantes que lo perciben. Menos dificultades presentan a la hora de captar la simultaneidad como yuxtaposición de un hecho que transcurre en el mismo tiempo (pregunta 4).

\section{Tabla 10}

Nivel de rendimiento máximo: simultaneidad

\begin{tabular}{|c|c|c|c|c|c|c|c|c|}
\hline & & Pregunta.3 & Pregunta.4 & Pregunta.5 & Pregunta.9 & Preugnta.10 & Media & $\begin{array}{l}\text { Nivel de rendimiento máximo } \\
\text { esperado }\end{array}$ \\
\hline $4^{\circ}$ de ESO & & $0 \%$ & $24,7 \%$ & $1,3 \%$ & $5,9 \%$ & $29,9 \%$ & $12,4 \%$ & Muy bajo \\
\hline $\begin{array}{l}2^{\circ} \\
\text { Bachillerato }\end{array}$ & de & $0 \%$ & $40,7 \%$ & $5,1 \%$ & $5,1 \%$ & $37,3 \%$ & $17,6 \%$ & Muy bajo \\
\hline
\end{tabular}

\subsection{La causalidad}

La causalidad (ver Tabla 11) se ha trabajado a partir de tres descriptores. En primer lugar, se ha analizado la causalidad como resultado de la duración (pregunta 5); la causalidad como consecuencia de un cambio (pregunta 8) y la causalidad a partir de explicaciones libres de tópicos (pregunta 10). En lo general, y sobre todo en Bachillerato, los estudiantes justifican ciertos hechos históricos a partir de causas concretas. No obstante, esta causalidad, muchas veces va ligada a explicaciones con tópicos o prejuicios históricos (pregunta 10).

\section{Tabla 11}

Nivel de rendimiento máximo: causalidad

\begin{tabular}{llllll}
\hline & Pregunta.5 & Pregunta.8 & Pregunta.10 & Media & Nivel de rendimiento máximo esperado \\
\hline $4^{\circ}$ de ESO & $28,6 \%$ & $39,6 \%$ & $13,6 \%$ & $27,3 \%$ & Bajo \\
2o de Bachillerato & $64,4 \%$ & $61 \%$ & $20,3 \%$ & $48,6 \%$ & Moderado \\
\hline
\end{tabular}




\subsection{La duración}

La duración (ver Tabla 12) se ha analizado como resultado de una repetición especifica de acontecimientos y/o años (pregunta 5). Dando unos resultados óptimos, sin embargo, la mayoría tienen una visión cronológica de la duración, donde en función de los años encuadran los hechos en corta, media o larga duración.

\section{Tabla 12}

Nivel de rendimiento máximo: duración

\begin{tabular}{lll}
\hline & Pregunta .5 & Nivel de rendimiento máximo esperado \\
\hline $4^{\circ}$ de ESO & $30,5 \%$ & Bajo \\
$2^{\circ}$ de Bachillerato & $64,4 \%$ & Bueno \\
\hline
\end{tabular}

\subsection{Comparación de los rendimientos máximos}

Si nos centramos en el rendimiento máximo, respecto al uso de las categorías temporales a modo general, vemos como de las 10 categorías analizadas, la periodización tiene un nivel de rendimiento máximo moderado en $4^{\circ}$ de la ESO y alto en $2^{\circ}$ de Bachillerato. El resto de las categorías temporales tienen unos niveles de rendimiento máximo que ronda del $10 \%$ al $40 \%$ aproximadamente (ver Gráfico.2). A excepción del cambio y continuidad y causalidad en $2^{\circ}$ de Bachillerato.

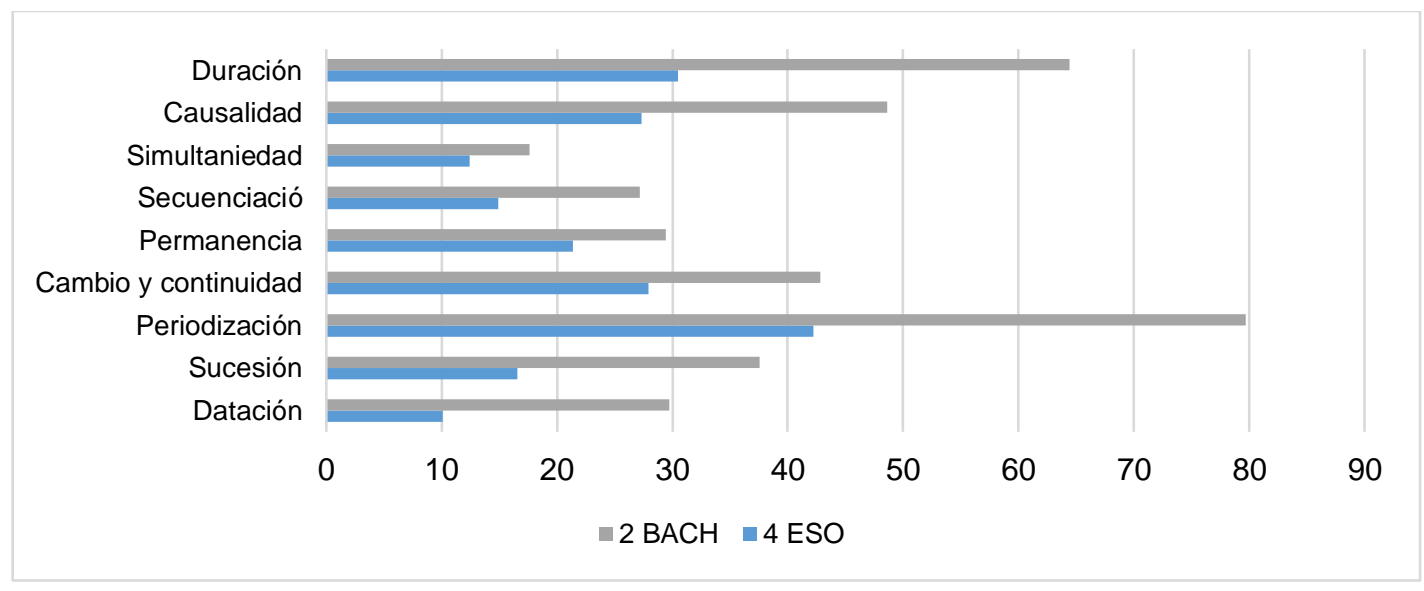

\section{Gráfico 2. Niveles de rendimiento máximo de los OTC}

Si nos centramos en los porcentajes del nivel de rendimiento máximo de cada una (ver Tabla. 13) de los operadores temporales analizados y hacemos una media aritmética global, comprobamos como en $4^{\circ}$ de la ESO tienen un promedio del $22,37 \%$, lo que se clasificaría como rendimiento del máximo esperado bajo. Los y las estudiantes de 2 o de Bachillerato, por su parte, tienen un promedio del $41,88 \%$, y por tanto un nivel de rendimiento del máximo esperado moderado.

\section{Tabla 13}

Nivel máximo de rendimiento de cada uno de los OTC

\begin{tabular}{|c|c|c|c|c|c|c|c|c|c|}
\hline & Datación & Sucesión & Periodización & $\begin{array}{l}\text { Cambio } \\
\text { continuidad }\end{array}$ & Permanencia & Secuenciación & Simultaneidad & Causalidad & Duración \\
\hline $\begin{array}{l}4^{\circ} \mathrm{ESO} \\
2^{\circ} \mathrm{BACH}\end{array}$ & $\begin{array}{l}\text { Muy bajo } \\
(10,1 \%) \\
\text { Bajo } \\
(29,7 \%)\end{array}$ & $\begin{array}{l}\text { Muy bajo } \\
(16,55 \%) \\
\text { Bajo } \\
(37,55 \%)\end{array}$ & $\begin{array}{l}\text { Moderado } \\
(42,2 \%) \\
\text { Bueno } \\
(79,7 \%)\end{array}$ & $\begin{array}{l}\text { Bajo } \\
(27,9 \%) \\
\text { Moderado } \\
(42,8 \%)\end{array}$ & $\begin{array}{l}\text { Bajo } \\
(21,4 \%) \\
\text { Bajo } \\
(29,4 \%)\end{array}$ & $\begin{array}{l}\text { Muy bajo } \\
(14,9 \%) \\
\text { Bajo } \\
(27,2 \%)\end{array}$ & $\begin{array}{l}\text { Muy bajo } \\
(12,4 \%) \\
\text { Muy bajo } \\
(17,6 \%)\end{array}$ & $\begin{array}{l}\text { Bajo } \\
(27,3 \%) \\
\text { Moderado } \\
(48,6 \%)\end{array}$ & $\begin{array}{l}\text { Bajo } \\
(30,5 \%) \\
\text { Bueno } \\
(64,4 \%)\end{array}$ \\
\hline
\end{tabular}




\section{Discusión de los resultados}

El presente estudio, que tenía como objetivo analizar los niveles de rendimiento máximo de estudiantes de $4^{\circ}$ de la ESO y $2^{\circ}$ de Bachillerato, muestra, tal como se ha percibido en otros estudios (De Groot-Reuvekamp, Ros, Van-Boxtel y Oort, 2015; Solé, 2019), que los y las estudiantes de cursos superiores tienen un mayor rendimiento en el uso de las categorías temporales. Además, nuestro estudio refleja como las categorías temporales de carácter cronológico (ej. la datación o la secuenciación) son las que presentan mayor dificultad (ver Figura 2).

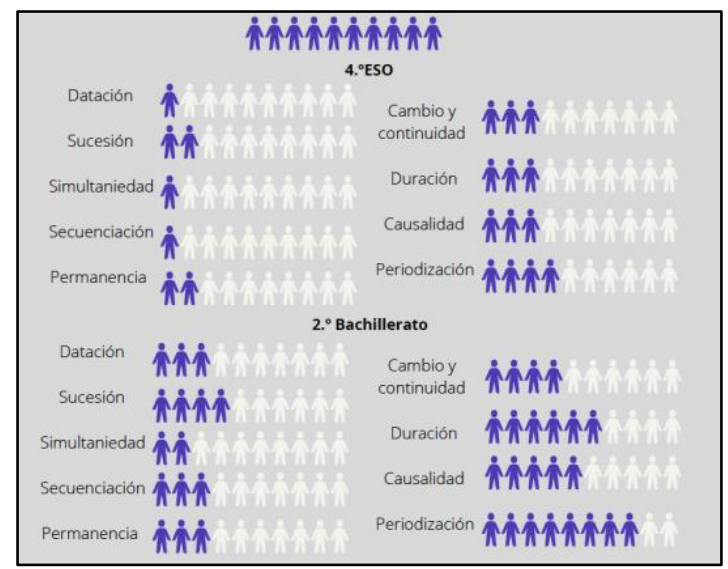

Figura 2. Nivel máximo de rendimiento x10 estudiantes

Estos resultados coinciden con el estudio Barbosa (2018) y con el de Llusà (2015). Éste último determinó que, para un óptimo desarrollo de los operadores temporales considerados complejos (ejemplo de ello, serían la duración, la continuidad, permanencia, etc.), resulta necesario dominar previamente las categorías cronológicas básicas. Además, el investigador concluyó que la datación es posiblemente una de las categorías temporales más difícil de adquirir. Sin embargo, esta diferenciación entre dos bloques de las categorías temporales no implica que se tenga que impartir los conceptos cronológicos como un elemento externo de las categorías conceptuales, sino más bien todo lo contrario. Como se expone en diversas investigaciones del área (De Groot-Reuvekamp, Ros y Van-Boxtel, 2017; Solé, 2019), las categorías temporales deberían introducirse cuando los discentes puedan darle un sentido dentro de la narrativa histórica. La explicación del concepto tiene que ir de la mano de la delimitación cronológica del período o suceso histórico trabajado en el aula (Jordheim, 2019). Esta acción resulta sumamente necesaria, ya que, además, hemos observado como los y las estudiantes tienen dificultades en aquellas preguntas del instrumento diseñado que deben aclarar, comprender o interpretar información en base al conocimiento previo (Sáiz, 2011;). Según dichos estudios esta tipología de actividad tiene una presencia moderada en los libros de texto de la educación secundaria (4ํㅡㄴ

Esta constatación hace replantearnos aspectos básicos de la didáctica del tiempo, ya que más que hablar de distintos niveles de dificultad de las categorías temporales tendríamos que hablar de grupos de categorías (en esta investigación las hemos denominado como cronológicas y conceptuales). Todas ellas deberían tener una presencia amplia en el currículo educativo, ya sea en Educación Infantil, Secundaria o Bachillerato, ya que son sumamente necesarias en el análisis de la temporalidad inmediata en Educación Infantil (Birkeland, 2018), la temporalidad cronológica en Educación Infantil y el ciclo inicial y medio de Educación Primaria (Birkeland, 2018; Solé, 2019), y la temporalidad histórica en el ciclo superior de Educación Primaria (De GrootReuvekamp, Ros y Van-Boxtel, 2017) y Educación Secundaria (De la Paz et al, 2017).

Además, los resultados del presente estudio ponen en discusión los niveles de dificultad de las categorías temporales presentadas por Blanco (2007) en su investigación sobre la temporalidad en los libros de texto. Blanco (2007) considera la datación, la sucesión y la simultaneidad operadores de baja dificultad. Mientras que la permanencia, la secuenciación, la continuidad, la empatía y el cambio son considerados operadores de media dificultad. Y la periodización, la duración y la causalidad como operadores de alta dificultad.

Entonces, ¿realmente tenemos que jerarquizar por nivel de dificultad las diversas categorías temporales? Diversas investigaciones sobre el aprendizaje del tiempo han querido ordenar el nivel de dificultad de las 
diversas categorías a partir del análisis de recursos varios, como por ejemplo el libro de texto (Blanco 2007) o las narrativas de los estudiantes (Hammond, 2014; De la Paz et al, 2017), y así, marcar unas pautas claras al profesorado sobre cómo introducir cada categoría temporal según la edad cognitiva del estudiante. De hecho, Blanco (2007) en su investigación concluye que hay una falta de unanimidad en el establecimiento de los niveles de dificultad de las distintas categorías. No obstante, investigaciones como la presente, o la de Llusà (2015), chocan con sus resultados y proponen una versión de la didáctica del tiempo histórico más interconectada entre categorías cronológicas y conceptuales.

Finalmente, hay que comentar que los estudios del tiempo, entre los cuales el nuestro, sobre sus estructuras y operadores temporales reafirman que es un campo aún por descubrir. El tiempo como tal, aún no forma parte de la enseñanza de la historia y, como consecuencia, es más frecuente que los y las estudiantes adquieran la noción de temporalidad fuera del ámbito educativa formal (Pagès, 1989 citado en Santiesteban, 2017).

\section{Conclusiones y limitaciones del estudio}

De la presente investigación emergen, principalmente, tres grandes conclusiones. En primer lugar, el estudio, así como las demás investigaciones realizadas en la misma línea, muestra que el desarrollo competencial del tiempo histórico entre los y las estudiantes es una asignatura pendiente y afecta directamente a la didáctica de la historia en el aula. Los bajos o muy bajos rendimientos académicos sobre dominio de los operadores temporales están estrechamente relacionados con un planteamiento histórico-didáctico que no está favoreciendo entrelazar entre ellas las explicaciones sobre los acontecimientos ocurridos en el pasado para una comprensión narrativa, continuum, del hecho histórico, factuum. Si bien los operadores temporales, permiten desglosar en varios descriptores el desarrollo de la competencia temporal, estos tienen que trabajarse a la vez que se estudian y se construye la comprensión del pasado, para promover la creación de nexos históricamente significativos. En este proceso, el dominio del tiempo histórico no puede ser un condicionante para la comprensión histórica, ni un contenido que vaya aparte del proceso de aprendizaje, sino más bien parte de la construcción narrativa causal. Es decir, que, si estudiamos el Imperio Romano como un periodo de larga duración, con efectos culturalmente significativos hasta nuestro presente, se puede llegar a producir una comprensión significativa incluso del aprendizaje de las categorías temporales cronológicas que tanto cuestan de memorizar. Y al revés, si entendemos, por ejemplo, conceptos como oligarquía, absolutismo, totalitarismos e, incluso, poder militar como decisiones coyunturales, los y las estudiantes pueden llegar a entender la simultaneidad espacial y temporal como una categoría conceptual no necesariamente cronológica. Así, podrán enriquecer su entendimiento del devenir histórico en términos de poder y organización política como un todo único diferenciado por etapas y circunstancias.

En segundo lugar, si nos atenemos a los resultados de nuestro estudio, se ha señalado, en acuerdo con otros autores, que la competencia temporal en relación con el dominio de los operadores temporales, tanto los más cronológicos, como los más conceptuales, aumenta en $2^{\circ}$ de bachillerato. Sin embargo, por cuanto decisiva, es esta mejora, no es dada por un aprendizaje más completo del tiempo, como demuestra nuestra discusión de los resultados, sino más bien, podría ser circunstancial al nivel de madurez cognitiva de estudiantes de mayor edad, y, posiblemente dada por la presión del examen final de acceso a la universidad, excesivamente basado en el factuum cronológico (Sáiz y Fuster, 2014).

En tercer y último lugar, como han marcado los resultados, vemos categorías temporales de carácter cronológicos, relativas al aprendizaje de hechos históricos, que tienen un nivel de rendimiento más bajo, a diferencia de algunos estudios y en línea con unos pocos. Sin embargo, eso no significa que el desempeño de las categorías más conceptuales sea significativamente más alto, sino, que los alumnos tienen al alcance más recursos para poder entender la continuidad y el cambio. Esto explicaría el número significativo de explicaciones aproximativas de los y las estudiantes, basadas en juicios personales de naturaleza presentista. Asimismo, queremos comentar, las limitaciones que se advierten en la investigación presentada. Por un lado, como se ha indicado anteriormente, la base de los análisis es la consideración de los rendimientos máximos. Sin duda alguna, representa un indicador interesante y necesario, pero a su vez implica no contemplar todos aquellos rendimientos medios que también son indicadores significativos. De esta manera, los rendimientos analizados de la muestra estudiada tienen un carácter orientativo y es necesario que sean contextualizados y confrontados con investigaciones previas, problematizando un ámbito esencial y emergente para el aprendizaje histórico: la didáctica del tiempo. Por otro lado, somos conscientes que no todas las categorías temporales han tenido el mismo número de descriptores (por ejemplo; la simultaneidad tiene 5 mientras que 
la secuenciación 1). Por tanto, el resultado emergente de cada una de ellas va sujeto a la significación del descriptor.

A pesar de ello, consideramos que este estudio ofrece puntos de interés en la línea de pesquisa sobre la didáctica del tiempo histórico en cuanto, al señalar la necesidad de una mayor y mejor consideración de la competencia temporal en el aprendizaje de la historia.

\section{Apoyos}

El desarrollo del presente artículo ha sido posible gracias al grupo de investigación y innovación DHIGECS, y se inscribe en el marco del proyecto estatal I+D+i (EDU 2015-65621-C3-3-R) "Desarrollo de la formación sociopolítica para una ciudadanía democrática: diseñó e implementación de materiales didácticos en ciencias sociales”.

\section{Referencias}

Assmann, A. (2019). A Creed That Has Lost its Believers? Reconfiguring the Concepts of Time and History. En M. Tammm \& L. Oliver (ed.). Rethinking Historial Time. Bloomsbury

Barbosa, L. J. (2018). Representaciones del tiempo histórico en un grupo de adolescentes de una institución pública peruana. [Trabajo Final de Grao, Pontificia Universidad Católica del Perú]. Recuperado de https://cutt.ly/ulyes34

Bellatti, I. (2019). La distribución espacio/temporal de los contenidos de los libros de texto en la educación secundaria y su implicación cultural en la construcción del relato histórico. Enseñanza de las Ciencias Sociales, 18, 17-33, https://raco.cat/index.php/EnsenanzaCS/article/view/363062

Bellatti, I., \& Sabido-Codina, J. (2017). La multidimensionalidad espacial y temporal como concepto substantivo en la enseñanza. CIMIE17-Investigación e innovación responsable. Bilbao: AMIE. Recuperado de https://cutt.ly/ITLiSOe

Birkeland, $\AA$. (2019). Temporal settings in kindergarten: a lens to trace historical and current cultural formation ideals? European Early Childhood Education Research Journal, 27(1), 53-67. https://doi.org/10.1080/1350293X.2018.1556534

Blanco, A. (2008). La representación del tiempo histórico en los libros de texto de primero y segundo de la enseñanza secundaria obligatoria. Enseñanza de las Ciencias Sociales, no7. Recuperado de https://cutt.ly/TTLi2IG

Blanco. A. (2007). La representación del tiempo histórico en los libros de texto de primero y segundo de la enseñanza secundaria obligatoria. Tesis doctoral: Universidad de Barcelona. Recuperado de https://cutt.ly/oTLkylh

Buck, T.M. (2020). Memoria, Contuitus, Exspectatio Zur zeitlichen Erstreckung des menschlichen Geistes. Sechter Workshop des Arbeitskreises Geschichtsdidaktik theoretisch. 02-03.03.2020, Universität zu Berlin.

Carretero, M., \& Montanero, M. (2008). Enseñanza y aprendizaje de la Historia: aspectos cognitivos y culturales. Cultura y educación, 20(2), 133-142. https://doi.org/10.1174/113564008784490361

De Groot-Reuvekamp, M., Ros, A., Van Boxtel, C., \& Oort, F. (2017). Primary school pupils' performances in understanding historical time. Education 3-13, 45(2), 227-242. https://doi.org/10.1080/03004279.2015.1075053

De Groot-Reuvekamp, M., Ros, A., \& Van Boxtel, C. (2018). Improving elementary school students' understanding of historical time: Effects of teaching with "Timewise". Theory \& Research in Social Education, 46(1), 35-67. https://doi.org/10.1080/00933104.2017.1357058

De la Paz, S., Montse-Sano, C., Felton, M., Croninger, R., Jackson, C., \& Worland-Piantedosi, K. (2017). A Historical Writing Apprenticeship for Adolescents: Integrating Disciplinary Learning with cognitive strategies. Reading Research Quarterly, 0 (0), 1-22. https://doi.org/10.1002/RRQ.147

Díaz-Barriga, F., García, J.-A., \& Toral, P. (2008). La comprensión de la noción de tiempo histórico en estudiantes mexicanos de primaria y bachillerato. Cultura y Educación, 20(2), $143-160$. https://doi.org/10.1174/113564008784490352

Duquette, C. (2011). Le rapport entre la pensee historique et la conscience historique. Elaboration d'un modèle d'interaction lors de l'apprentissage de l'histoire chez les élèves de cinquième secondaire des écoles francophones du Québec. Université Laval Québec.

Hammond, K. (2014). The knowledge that 'flavours' a claim: towards building and assessing historical knowledge on three scales. Teaching History, (157), 18. Recuperado de https://cutt.ly/9TLkSvK

Jacott, L. (1995). La comprensión de la causalidad histórica. Tesis doctoral: Universidad Autónoma de Madrid.

Jordheim, H. (2019). Return to Chronology. En M. Tammm \& L. Oliver (ed.). Rethinking Historial Time. Bloomsbury

Keynes, M. (2021). Engaging transitional justice in Australian history curriculum: Times, temporalities and historical thinking. Curriculum Inquiry, 51(4), 413-436. https://doi.org/10.1080/03626784.2021.1938972

Lee, P., \& Shemilt, D. (2004). 'I just wish we could go back in the past and find out what really happened': Progression in understanding about historical accounts. Teaching History, (117), 25. Recuperado de https://cutt.ly/BTLk4Ts

Löfgren, K. (2017, julio 9). Nominal dichotomous yes/no data: Krippendorff aplha inter-raer reliability . [Vídeo]. Recuperado de https://www.youtube.com/watch?v=NcC99TrynKQ

López, P. L. (2004). Población muestra y muestreo. Punto cero, 9(08), 69-74. Recuperado https://cutt.ly/cTLlopS

López, A., \& Llull, J. (2017). Un análisis de los recursos didácticos empleados para el aprendizaje del tiempo histórico en los libros de texto de Educación Primaria. Didáctica de las ciencias experimentales y sociales, (32), 71-92. https://doi.org/10.7203/dces.32.9620 
Llusà, J. (2015). Ensenyar historia des de la contemporaneïtat estudi de cas sobre la construcción i aplicación del concepte contemporaneïtat a l'ESO. Tesis doctoral: Universidad Autònoma de Barcelona. Recuperado de https://cutt.ly/MTLIxxG

Mattozzi, I. (2004). Enseñar a escribir sobre la Historia. Enseñanza de las Ciencias Sociales, 3, 39-48. Recuperado de https://cutt.ly/hTLIKc5

Mattozzi, I. (1988). I bambini, il tempo, la storia: educazione temporale e curricolo di storia nella scuola elementale. CIDI di Firenze (dir.). Tempo e spazio, dimensioni del sapere. Dalle hipótesis teoriche alle practique didattiche. Mondadori.

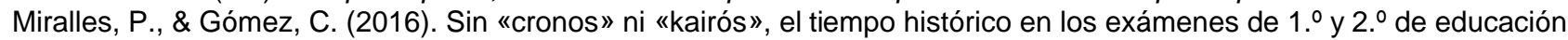
secundaria obligatoria. Enseñanza de las Ciencias Sociales. 15,15-26. DOI: 10.1344/ECCSS2016.15.2

Ost, F. (2000). El tiempo, cuarta dimensión de los derechos humanos (Falcón, M.J, trad.). Anuario de Derechos Humanos. Nueva Época, (1), 287-310. (Obra original publicada en 1999). Recuperado de https://cutt.ly/CTLzalU

Pinto, D. C., \& Pagès, J. (2020). Los ritmos del cambio en la enseñanza-aprendizaje de la periodización. Una propuesta desde la historia global. Clio \& Asociados, (30), 90. Recuperado de https://cutt.ly/oTLzPSa

Sánchez-Carrión, J. J. (2012). La encuesta, herramienta cognitiva. Papers: revista de sociologia, 97(1), 0169-192. Recuperado de https://ddd.uab.cat/record/85224

Santisteban, A. (2017). Del tiempo histórico a la conciencia histórica: cambios en la enseñanza y el aprendizaje de la historia en los últimos 25 años. Diálogo andino, (53), 87-99. Recuperado de https://cutt.ly/NTLzJvg

Sáiz, J. (2011). Actividades de libros de texto de Historia, competencias básicas y destrezas cognitivas, una difícil relación: análisis de manuales de $1^{\circ}$ y $2^{\circ}$ de ESO. Revista Didáctica de las Ciencias Experimentales y Sociales, $25,37-64$. Recuperado de https://roderic.uv.es/handle/10550/21329

Sáiz, J. (2013). Alfabetización histórica y competencias básicas en libros de texto de historia y en aprendizajes de estudiantes. Revista didáctica de las ciencias experimentales y sociales, 27, 43-66. Recuperado de https://roderic.uv.es/handle/10550/34573

Sáiz, J., \& Colomer, J.C. (2014). ¿Se enseña pensamiento histórico en libros de texto de Educación Primaria?. Clio. History and History teaching, 40. Recuperado de https://cutt.ly/0E4YQZ1

Sáiz, J. \& Fuster, C. (2014). Memorizar historia sin aprender pensamiento histórico: Las Pau de historia de España. Investigación en la escuela, 84, 47-58. ttp://hdl.handle.net/11441/59755.

Scharager, J., \& Armijo, I. (2001) Metodología de la Investigación para las Ciencias Sociales [CD-ROM]. Escuela de Psicología, SECICO Pontificia Universidad Católica de Chile.

Solé, G. (2019). Children's understanding of time: A study in a primary history classroom. History Education Research Journal, 16(1), 158-73. https://doi.org/10.18546/HERJ.16.1.13

Seixas, P. \& Morton, T. (2012). The Big Six. Historical Thinking Concepts. Nelson, 2012.

Strauss, A., \& Corbin, J. (2002). Bases de la investigación cualitativa. Técnicas y procedimientos para desarrollar la teoría

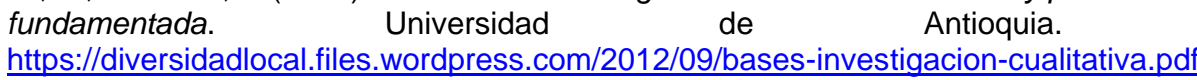
$\underline{\text { https://diversidadlocal.files.wordpress.com/2012/09/bases-inves }}$

Trepat, C.A. (2002). El tiempo en la didáctica de las ciencias sociales. En Trepat, C.A., \& Comes, P. El tiempo y el espacio en la didáctica de las ciencias sociales, 7-122. Graó.

Torres, P.A. (2001). Enseñanza del tiempo histórico. Ediciones de la Torre.

Torres, J. J., \& Perera, V. H. (2010). Cálculo de la fiabilidad y concordancia entre codificadores de un sistema de categorías para el estudio del foro online en e-learning. Revista De Investigación Educativa, 27(1), 89-103. Recuperado de https://revistas.um.es/rie/article/view/94291

Wilschut, A. (2019). Historical consciousness of time and its societal uses. Journal of Curriculum Studies, 51(6), 831-849. https://doi.org/10.1080/00220272.2019.1652939 